\title{
Organic molecules in protostellar environments
}

\author{
Cecilia Ceccarelli \\ Laboratoire d'Astrophysique de Grenoble, Université Joseph Fourier, UMR 5571-CNRS, \\ France \\ email: Cecilia.Ceccarelli@obs.ujf-grenoble.fr
}

\begin{abstract}
The sequence that brings matter from a molecular cloud to a fully developed star plus planetary system seems to be a unique and rich chemistry laboratory where, step by step, molecular complexity increases. During the cold pre-collapse phase, atoms and simple molecules, like $\mathrm{CO}$, freeze out onto the dust grains, forming icy mantles. Reactions on the grain surfaces likely form hydrogenated molecules (notably $\mathrm{H}_{2} \mathrm{O}, \mathrm{CH}_{4}, \mathrm{H}_{2} \mathrm{CO}, \mathrm{CH}_{3} \mathrm{OH}$, and $\mathrm{NH}_{3}$ ) and perhaps even more complex organic molecules. The hallmark of this era is the super-deuteration phenomenon, i. e. the abnormal enhancement of molecules containing one or more D atoms instead of $\mathrm{H}$ atoms, by up to 13 orders of magnitude with respect to the cosmic elemental $\mathrm{D} / \mathrm{H}$ ratio $\left(\sim 10^{-5}\right)$. The frozen molecules are released into the gas upon warming by the forming star and undergo reactions which further increase the molecular complexity, leading to several complex organic molecules. Products of this efficient chemical factory are observed in the hot corinos, which are warm $(\sim 100 \mathrm{~K})$, dense $\left(\sim 10^{7}-10^{8} \mathrm{~cm}^{-3}\right)$ solar-system-sized regions at the centre of the collapsing envelopes of solar type protostars. In this contribution, I review what is known about the organic molecules in protostellar environments, with emphasis on the hot corinos, and how possibly the organic molecules formed at this stage may constitute an heritage for the forming planetary system.
\end{abstract}

Keywords. Astrochemistry, stars: formation

\section{Introduction}

In the last two decades, the number of molecules detected in space has steadily augmented with time and it is now larger than 100 (see for example the census in http://www.cv.nrao.edu/ awootten/allmols.html). In addition to the number of discovered molecules, also the number of atoms composing them has augmented, reaching more than 10. Polyatomic molecules seem to be, therefore, common in space, despite the cold, almost void and harsh conditions. Among the general class of polyatomic molecules, Complex Organic Molecules (hereinafter called COMs) are of particular interest because they are potentially connected to the chemistry of life, as known on Earth, by their own definition (Organic). Note that in this contribution the adjective Complex refers to molecules containing 4 or more atoms (only), namely much simpler molecules than those that normal chemists would call complex. Formaldehyde $\left(\mathrm{H}_{2} \mathrm{CO}\right)$, for example, is a COMs in our definition while it would hardly be considered complex in another context.

Although found in several different regions, undoubtedly the "retail shops" of COMs are where stars form, the Star Formation Regions. COMs are found in a large variety and quantity in Star Forming Regions, be them nurseries of low or high mass stars. One the newest results of these last years studies is the understanding that COMs form, evolve and become more complex all along the birth process of the star and planetary system. In few words, the history of COMs is tightly linked to the history of star formation, from 


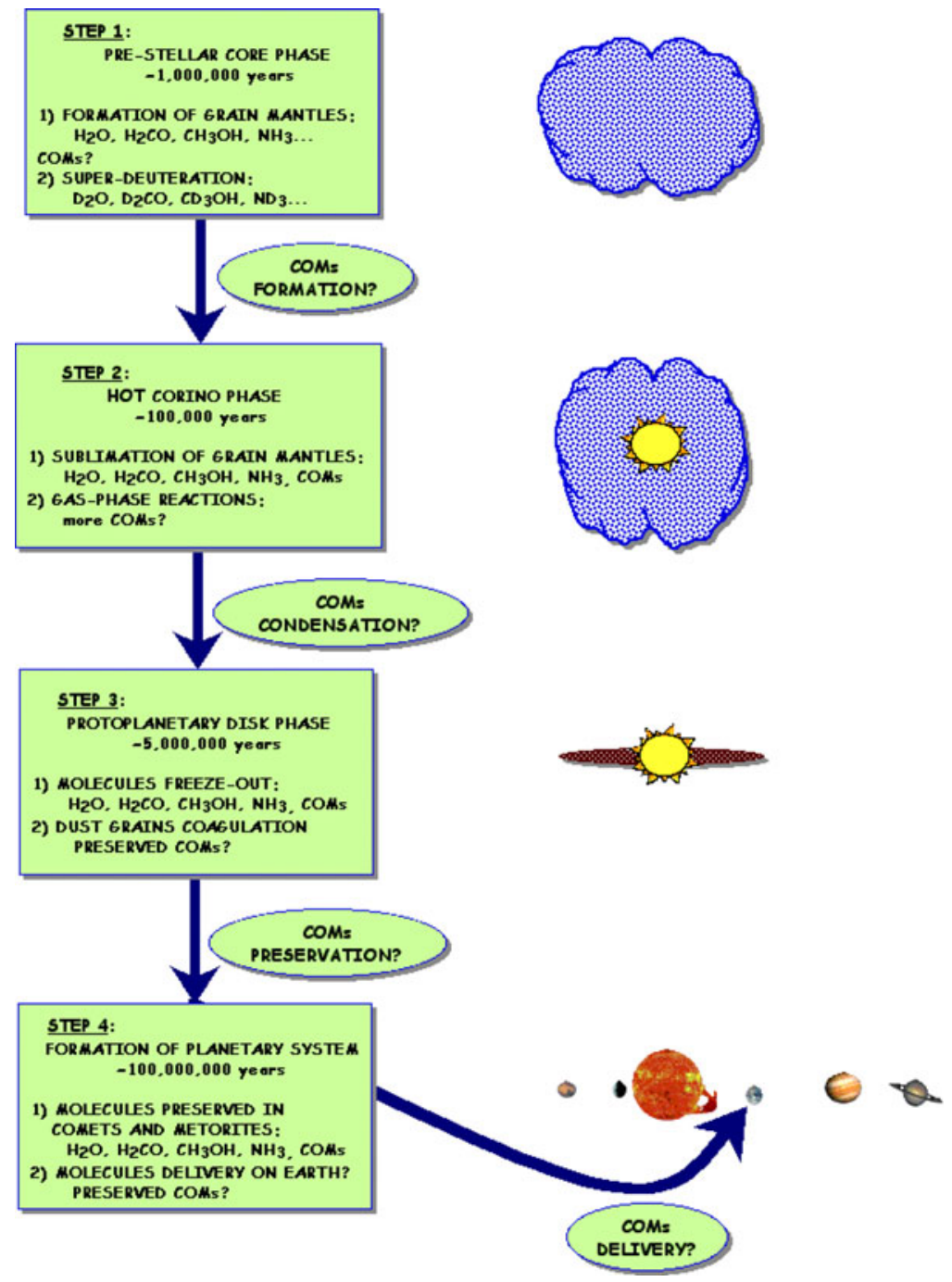

Figure 1. Scheme of the interconnection between the history of a Sun-like star birth and the COMs formation.

the very fist phases of incipient collapse to the final steps towards planet formation. This review will give a flavor of this fascinating inter-connected history. A complementary review can be found in Ceccarelli et al. (2007), among other reviews of the subject.

\section{The history of Complex Organic Molecules during Star Formation}

Figure 1 shows schematically the interconnection between the process of a Sun-like star formation and the formation, evolution and conservation of COMs. The history can be divided into four major periods: Pre-Stellar Core, Hot Corino, Proto-Planetary Disk and Planet Formation periods.

\subsection{Pre-Stellar Core period}

This is the phase just before the collapse begins. For about $10^{6}$ years, matter slowly accumulates towards the center of the condensation from which a star plus planetary system 
will ultimately form. There are still many uncertainties how exactly this accumulation process proceeds and what triggers it (see the discussion in Ward-Thompson et al. (2007) and Ballesteros-Paredes et al. (2007) for recent reviews). However, it is clear that at the center of the Pre-Stellar Cores the temperature drops to less than $10 \mathrm{~K}$ and the density reaches more than $10^{5} \mathrm{~cm}^{-3}$. In these conditions, all heavy-element bearing molecules freeze-out onto the dust grains, enveloping the grains by icy mantles. Moreover, once stuck on the grain surfaces $\mathrm{CO}$ molecules are hydrogenated and oxygenated forming the "first-generation" COMs: formaldehyde $\left(\mathrm{H}_{2} \mathrm{CO}\right)$, methanol $\left(\mathrm{CH}_{3} \mathrm{OH}\right)$, and perhaps other more complex COMs like formic acid ( $\mathrm{HCOOH})$. While the formation of formaldehyde and methanol during this period is supported by direct observations of these molecules in Pre-Stellar Cores (Bacmann et al. 2003), the formation of other COMs is, at present, more speculative (Bottinelli et al. 2007). Simultaneously with the CO freezing and triggered by that, a peculiar deuterium chemistry takes over leading to the super-deuteration phenomenon (Bacmann et al. 2003, Roberts et al. 2003, Butner et al. 2007). Plenty of Dbearing molecules are formed, whose abundance with respect to the relevant H-bearing molecules can be enhanced by up to 13 orders of magnitude the elemental $\mathrm{D} / \mathrm{H}$ ratio (Bacmann et al. 2003, Caselli et al. 2003, Crapsi et al. 2005, Ceccarelli et al. 2007) †. The super-deuteration is the hallmark of this period, the Arianna's thread which links all the various phases from the Pre-Stellar Cores to the planet, comets and meteorites in formed planetary systems, as discussed below.

\subsection{Hot Corino period}

Once the collapse starts, matters falls inward and feeds the newly born star. The gravitational energy is released into radiation, and the matter at the center of the infalling envelope warms up (Ceccarelli et al. 1996). Where the dust temperature is larger than about $100 \mathrm{~K}$, the grain mantles formed during the previous period are sublimated and all their components are injected into the gas phase. These regions are called Hot Corinos (Ceccarelli 2004, Bottinelli et al. 2004b). Therefore, the COMs formed in the Pre-Stellar Core period are now found in the gas phase. In addition to them, other COMs may be formed during the Hot Corino period either on the warm grain surfaces thanks to the increased mobility of atoms and molecular groups (Garrod \& Herbst 2006, Garrod et al. 2008) or in the gas phase thanks to reactions with activation barriers which become efficient at temperatures larger than $\sim 100 \mathrm{~K}$ (Millar et al. 1991). At present, it is not clear which one of the various mentioned processes contribute to the formation of what COMs. Indirect evidences suggest that molecules like formic acid, methyl formate and dimethyl ether are synthesized on the grain surfaces, possibly already at the epoch of the Pre-Stellar Core (Requena-Torres et al. 2006, Requena-Torres et al. 2007, Bisschop et al. 2007, Bottinelli et al. 2007). One key information, in this respect, is the degree of deuteration of COMs. So far we have observations of only deuterated formaldehyde (singly and doubly deuterated: Ceccarelli et al. 1998, Parise et al. 2006) and methanol (singly, doubly and triply deuterated: Parise et al. (2002), Parise et al. (2004) in Hot Corinos. Both molecules have a huge enhancement of the $\mathrm{D} / \mathrm{H}$ ratio, $\sim 30 \%$ in the singly deuterated forms, $\sim 10 \%$ in the doubly deuterated forms and $\sim 5 \%$ in the triply deuterated form, pointing to a formation in the Pre-Stellar Core period. Other molecules like $\mathrm{CH}_{3} \mathrm{CN}$ could be gas phase products (Bottinelli et al. 2007).

One important point to mention is that COMs have been detected in all Hot Corinos so far observed. Table 1 summarizes the observed COMs and the Hot Corinos where they

$\dagger$ Note that the bulk of deuterium is in HD molecules and that the super-deuteration only involves trace, low abundance molecules which take up likely only a fraction of the elemental deuterium. 
were observed at the date when this review has been written. We have to emphasize that the listed molecules do not reflect a systematic search of COMs, so that many others could be present and just not searched for so far (see next section).

\begin{tabular}{|c|c|c|}
\hline Hot Corino & | Detected COMs & |References \\
\hline IRAS16293-2422 & $\begin{array}{l}\mathrm{HCOOH}, \mathrm{CH}_{3} \mathrm{CHO}, \mathrm{CH}_{3} \mathrm{OCHO} \\
\mathrm{CH}_{3} \mathrm{OCH}_{3}, \mathrm{HCOOCH} 3, \mathrm{CH}_{3} \mathrm{CCH} \\
\mathrm{CH}_{3} \mathrm{CN}, \mathrm{C}_{2} \mathrm{H}_{5} \mathrm{CN}\end{array}$ & $1,2,34,5$ \\
\hline NGC1333-IRAS4A & $\mathrm{HCOOH}, \mathrm{HCOOCH}_{3}, \mathrm{CH}_{3} \mathrm{CN}$ & $6,7,8$ \\
\hline NGC1333-IRAS4B & $\mathrm{HCOOCH}_{3}, \mathrm{CH}_{3} \mathrm{CN}$ & 7,9 \\
\hline NGC1333-IRAS2A & $\mathrm{CH}_{3} \mathrm{OCH}_{3}, \mathrm{CH}_{3} \mathrm{CN}$ & 7,10 \\
\hline
\end{tabular}

Table 1. List of the detected COMs in Hot Corinos. References: 1- Cazaux et al. (2003); 2Kuan et al. (2004); 3- Bottinelli et al. (2004b); 4- Chandler et al. (2005); 5- Remijan \& Hollis (2006); 6- Bottinelli et al. (2004a); 7- Bottinelli et al. (2007); 8- Bottinelli et al. (2008); 9- Sakai et al. (2006); 10- Jørgensen et al. (2005).

\subsection{Proto-Planetary period}

With time, the envelope surrounding the central future star dissipates (both because matters falls into the future star and because a large fraction is spit out by energetic outflowing jets). What remains is a surrounding disk, called Proto-Planetary Disk. This is the last step towards planet formation. At the beginning, proto-planetary disks are composed by gas and dust in the same 100:1 portion than in molecular clouds: disks are said to be gas rich. With time, the gas is dissipated -because it falls towards the central star and because it is photo-dispersed (Dullemond et al. 2007). The disk becomes gas poor, and disks in this phase are called "debris disks" because mostly formed by dust grains. For reasons not yet clear, grains coagulate into large rocky bodies which will eventually become planets, comets and meteorites. A planetary system is finally formed. From the chemical point of view, this period is characterized by three processes: i) new COMs are formed, especially in the innermost warm and dense zones when the disk is still gas rich; ii) as the disk cools down, COMs freeze-out onto the dust grains, forming ice mantles enriched of the COMs; iii) the coagulated dust grains may trap some of these ices and conserve them as an heritage of the past eons.

The first process is now supported by recent observations that show large quantities of acetylene (Lahuis et al. 2006, Brittain et al. 2007), a potentially crucial molecule for the synthesis of more complex COMs. Agundez et al. (2008) argued that acetylene, as other hydrocarbons, is produced in the inner, warm and dense regions of the Proto-Planetary Disks thanks to the intense FUV illumination from the newly born star. The large densities and temperatures make that the destruction of $\mathrm{H}_{2} \mathrm{C}_{2}$ and other hydrocarbons by the FUV photons are balanced by their fast formation because of reactions with activation barriers inhibited at low temperatures, giving rise to a very peculiar rich organic chemistry. The reader is referred to the contribution by Henning \& Semenov in this volume for a further discussion on the organic molecules in Proto-Planetary Disks.

With increasing time, the accretion rate from the disk to the star would diminish and, consequently, the viscous heating of the disk would diminish too. The disk becomes "passive", namely only warmed by the inner star radiation, and slowly cools down. Whatever the origin and the moment of formation, any COM in the gas phase would end up to freeze-out onto the dust grains in the regions where the dust temperature is lower than about $100 \mathrm{~K}$. This limit is called snow-line because water remains frozen outwards of this 
line. Very likely, frozen COMs are also trapped in the ices forming a precious legacy for the planetary system objects, as discussed in the next paragraph.

\subsection{Planetary System period}

Although a clear theory of planet formation doesn't exist yet, planets form, and with them comets and meteorites, the debris of the planet formation process. Rocky planets, comets and meteorites must be made of the material in the original Proto-Planetary Disk. The exact process which coagulates the submicron-size dust grains into large km-size rocks is still a mystery, but in a way or another this coagulation must occur. Depending on how gentle the process is, the ices coating the grains may be conserved with their precious load of previously formed COMs, at least in part. It is possible that the conservation will be more efficient in the outer zones of the disk than in the inner ones, because of the likely lower turbulence, shocks etc. However, because large dust grains migrate inward and, once formed, giants planets migrate outwards causing a re-mixing of the planetary system bodies location, the pristine COMs-enriched ices may still be found into the inner, Earth-like planet forming zone. As a matter of fact, many evidences point to such a picture for the Solar System formation. Based on the measured deuterium enrichment, comets and meteorites seem to have inherited some of the pristine material (see the contributions by Alexander et al., Cody et al., and Mumma, in this volume). What COMs such a pristine material really contained is an open question. One way to answer the question is to go back to the COMs retail shops, the Hot Corinos, and obtain a census of the COMs present at that stage. Next section discuss how this is possible.

\section{Unbiased Spectral Surveys: the ultimate tool for COMs census}

Molecules are identified in space by their lines, either in emission or absorption, from rotational, ro-vibrational, bending or electronic transitions. For the conditions existing in the Hot Corinos, the rotational lines are the preferential, if not the only probe we have to identify molecules. In general, the larger the molecule the smaller the frequency of emission. Besides, the larger the molecule the larger the number of emitted lines, which implies that the energy is spread over several lines, making them relatively weak even when the molecule dipole moment is large. For this reason, the larger the molecule the more difficult results to identify it in space. Thus, having a complete census of COMs will be inevitably hampered by the intrinsic weakness of the lines and by the presence of a grass of weak lines emitted by large molecules. In practice, in order to identify large molecules few lines from the molecule are not enough because of the relatively high probability of line coincidence. In the case of large molecules, experience has taught that dozens of lines are necessary for ensure the identification (Snyder et al. 2005). The best way to obtain a census of large molecules is provided by the unbiased spectral surveys, namely the coverage of large frequency intervals. Several surveys of high mass Hot Cores exist covering the millimeter to sub-millimeter bands observable from ground (Helmich \& van Dishoeck 1997, Comito et al. 2005). In the case of hot corinos there is only one published study towards IRAS16293-2422 (Blake et al. 1994, van Dishoeck et al. 1995). The most recent and sensitive survey by Caux et al. (in prep.) $\dagger$ covers

$\dagger$ The members of the international Consortium which carried out the spectral survey of IRAS16293-2422 are: E. Caux, C. Vastel \& A. Walters (CESR Toulouse, France), A. Castets, C. Ceccarelli, C. Kahane, B. Lefloch \& A. Bacmann (LAOG Grenoble, France), V. Wakelam (LA3B Bordeaux, France), F. Helmich, X. Tielens \& S. Cazaux (SRON Groningen, NL), E. van Dishoeck \& S. Bisshop (Leiden Observatory, NL), P. Schilke, C. Comito \& B. Parise (MPIfA Bonn, Germany). 


\section{Rest Frequency $(\mathrm{GHz})$}

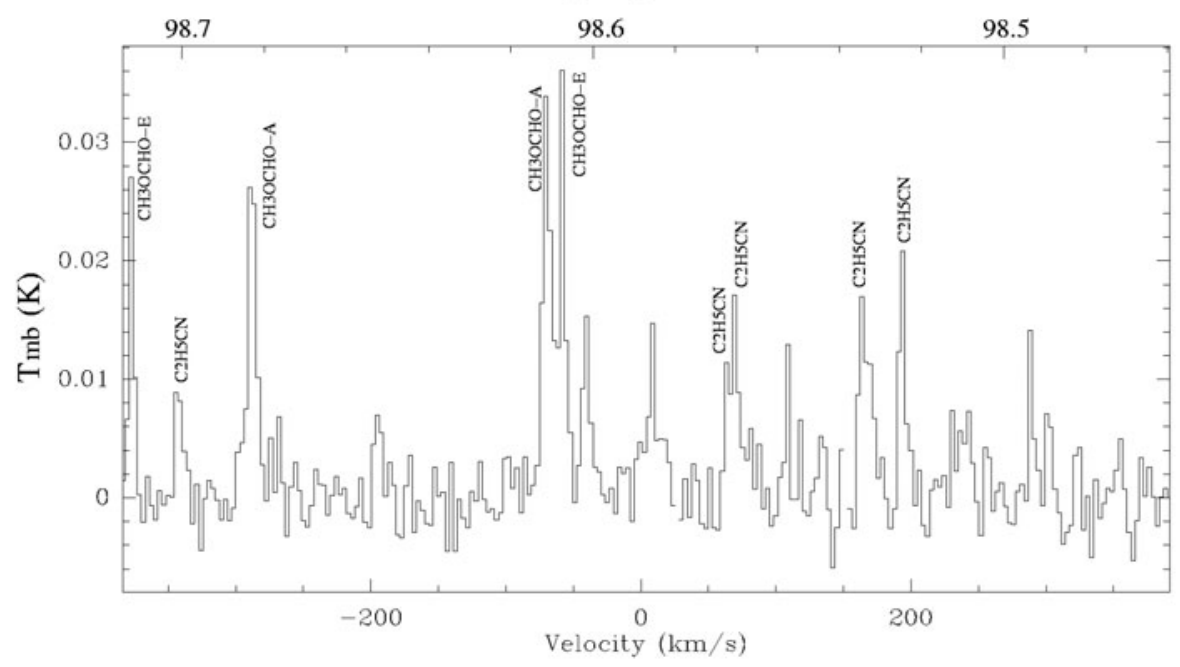

Rest Frequency $(\mathrm{GHz})$

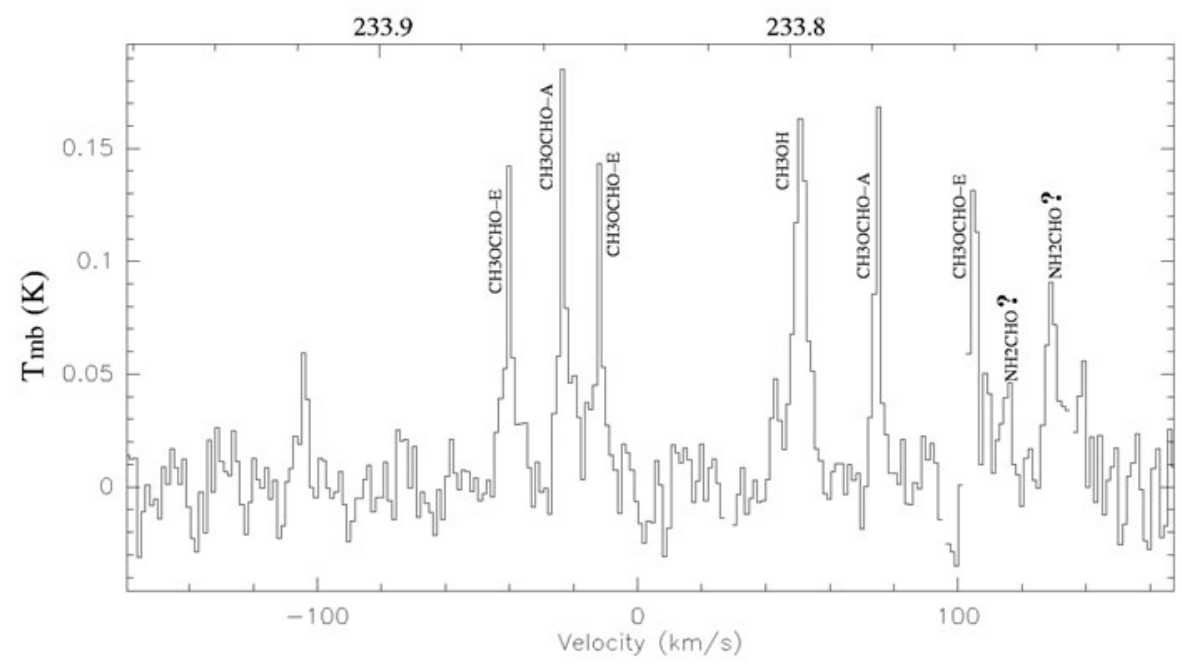

Figure 2. Sample spectra of the Hot Corino IRAS16293-2422 (courtesy by E. Caux).

the 3, 2, 1 and 0.8 millimeter bands with the largest existing telescopes: IRAM and JCMT respectively. Figure 2 shows a sample of this survey and the richness of the spectrum. For the first time, several COMs have been detected in a Hot Corino with dozens of lines. This not only permits to make sure identifications of the molecule, but also to derive reliable abundances in the outer envelope and Hot Corino of the source respectively. Here we report the example of propyne $\left(\mathrm{CH}_{3} \mathrm{CCH}\right)$, an hydrocarbon of the alkynes family, extremely reactive on Earth and a building block of more complex organic molecules. Figure 3 shows the rotational diagram of this molecules, with 44 detected lines. Cazaux et al. (2003) previously reported the detection of this molecule based on four lines and estimated an abundance in the Hot Corino of about $3 \times 10^{-7}$ with respect to $\mathrm{H}_{2}$. The one order larger number of detected lines in the new survey by Caux and colleagues allows to have a much more sophisticated analysis, where the contribution 


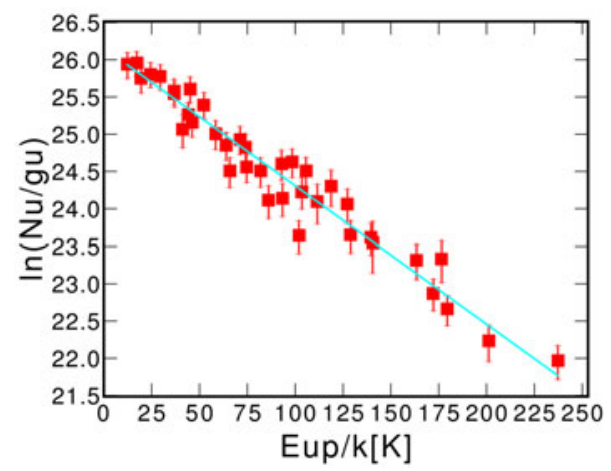

Figure 3. Rotational diagram of propyne in IRAS16293-2422 (from Caux et al., in preparation). The diagram reports the log column density of each rotational level as function of the energy of the level. Note that no beam dilution factor has been applied here to take into account for the different emitting sizes.

from the outer envelope and the Hot Corino can be disentangled. The comparison of the measured line fluxes with those predicted by a model that takes into account the density and temperature profiles derived by several previous observations (Ceccarelli et al. 2000) gives a propyne abundance of $\sim 3 \times 10^{-10}$ and $\sim 1 \times 10^{-7}$ in the outer and Hot Corino regions respectively (Ceccarelli et al., in preparation). Therefore, propyne is present in both regions, although its abundance is 300 times larger in the Hot Corino region. The relative abundance between the regions give us an hint on how this molecule is formed.

Finally, the systematic study of the abundance of COMs in the outer envelope and Hot Corino will likely allow to shed light on the mechanisms of formation and destruction of COMs in the Inter-Stellar Medium and, consequently, on the potential heritage passed throughout the various phases of the formation of a planetary system as the Solar System.

\section{Conclusions}

Despite the harsh conditions, low temperatures and densities, presence of FUV photons, shocks etc, Star Forming Regions are nurseries not only of stars but also of COMs. Several COMs have already been detected, but we know that many other await to be discovered. Very likely we have just seen the tip of the iceberg so far. Digging into the deep to search for the rest of the iceberg is one of the challenges of the next decade. We will be aided in this adventure by two new powerful telescopes: Herschel and ALMA. Herschel, a $3.5 \mathrm{mt}$ space telescope to be launched in 2009, will allow observations in regions of the spectrum mostly unexplored, between 500 and 2,000 GHz, because blocked by the Earth atmosphere (http://herschel.esac.esa.int/home.shtml). ALMA (http://www.eso.org/sci/ facilities/alma/), the 64 telescopes millimeter interferometer which will start operation in 2010, will provide unprecedent sensitivity observations, with a spatial resolution which will allow to image the COMs-rich regions. No doubts that next decade will give us the opportunity to answer some fundamental questions on COMs: what COMs are formed, where and why. In turn, this will also likely provide the answer whether Earth received the heritage of the ancient eons.

\section{References}

Agundez, M., Cernicharo, J., \& Goicoechea, J. R. 2008, A\& A, 483, 831

Bacmann, A., Lefloch, B., Ceccarelli, C., et al. 2003, ApJ (Letters), 585, L55 
Ballesteros-Paredes, J., Klessen, R. S., Mac Low, M.-M., \& Vazquez-Semadeni, E. 2007, in: B. Reipurth, D. Jewitt, \& K. Keil (eds.), Protostars and Planets V, p. 63

Bisschop, S. E., Jørgensen, J. K., van Dishoeck, E. F., \& de Wachter, E. B. M. 2007, A\&A, 465, 913

Blake, G. A., van Dishoek, E. F., Jansen, D. J., Groesbeck, T. D., \& Mundy, L. G. 1994, ApJ, 428,680

Bottinelli, S., Ceccarelli, C., Lefloch, B., et al. 2004a, ApJ, 615, 354

Bottinelli, S., Ceccarelli, C., Neri, R., et al. 2004b, ApJ (Letters), 617, L69

Bottinelli, S., Ceccarelli, C., Neri, R., \& Williams, J. P. 2008, ApJ (Letters), accepted

Bottinelli, S., Ceccarelli, C., Williams, J. P., \& Lefloch, B. 2007, A $\mathscr{E} A, 463,601$

Brittain, S. D., Simon, T., Najita, J. R., \& Rettig, T. W. 2007, ApJ, 659, 685

Butner, H. M., Charnley, S. B., Ceccarelli, C., et al. 2007, ApJ (Letters), 659, L137

Caselli, P., van der Tak, F. F. S., Ceccarelli, C., \& Bacmann, A. 2003, A\&A (Letters), 403, L37

Cazaux, S., Tielens, A. G. G. M., Ceccarelli, C., et al. 2003, ApJ (Letters), 593, L51

Ceccarelli, C. 2004, in Astronomical Society of the Pacific Conference Series, p. 195

Ceccarelli, C., Caselli, P., Herbst, E., Tielens, A. G. G. M., \& Caux, E. 2007, in: B. Reipurth,

D. Jewitt, \& K. Keil (eds.), Protostars and Planets V, p. 47

Ceccarelli, C., Castets, A., Caux, E., et al. 2000, A\&A, 355, 1129

Ceccarelli, C., Castets, A., Loinard, L., Caux, E., \& Tielens, A. G. G. M. 1998, A\&A (Letters), $338, \mathrm{~L} 43$

Ceccarelli, C., Hollenbach, D. J., \& Tielens, A. G. G. M. 1996, ApJ, 471, 400

Chandler, C. J., Brogan, C. L., Shirley, Y. L., \& Loinard, L. 2005, ApJ, 632, 371

Comito, C., Schilke, P., Phillips, T. G., et al. 2005, ApJS, 156, 127

Crapsi, A., Caselli, P., Walmsley, C. M., et al. 2005, ApJ, 619, 379

Dullemond, C. P., Hollenbach, D., Kamp, I., \& D'Alessio, P. 2007, in: B. Reipurth, D. Jewitt, $\&$ K. Keil (eds.), Protostars and Planets V, p. 555

Garrod, R. T. \& Herbst, E. 2006, A\& A, 457, 927

Garrod, R. T., Widicus Weaver, S. L., \& Herbst, E. 2008, ArXiv e-prints, 803

Helmich, F. P. \& van Dishoeck, E. F. 1997, A\&AS, 124, 205

Jørgensen, J. K., Bourke, T. L., Myers, P. C., et al. 2005, ApJ, 632, 973

Kuan, Y.-J., Huang, H.-C., Charnley, S. B., et al. 2004, ApJ (Letters), 616, L27

Lahuis, F., van Dishoeck, E. F., Boogert, A. C. A., et al. 2006, ApJ (Letters), 636, L145

Millar, T. J., Herbst, E., \& Charnley, S. B. 1991, ApJ, 369, 147

Parise, B., Castets, A., Herbst, E., et al. 2004, A\&A, 416, 159

Parise, B., Ceccarelli, C., Tielens, A. G. G. M., et al. 2006, A\&\&A, 453, 949

Parise, B., Ceccarelli, C., Tielens, A. G. G. M., et al. 2002, A\&SA (Letters), 393, L49

Remijan, A. J. \& Hollis, J. M. 2006, ApJ, 640, 842

Requena-Torres, M. A., Marcelino, N., Jiménez-Serra, I., et al. 2007, ApJ (Letters), 655, L37

Requena-Torres, M. A., Martín-Pintado, J., Rodríguez-Franco, A., et al. 2006, A 6 A, 455, 971

Roberts, H., Herbst, E., \& Millar, T. J. 2003, ApJ (Letters), 591, L41

Sakai, N., Sakai, T., \& Yamamoto, S. 2006, PASJ (Letters), 58, L15

Snyder, L. E., Lovas, F. J., Hollis, J. M., et al. 2005, ApJ, 619, 914

van Dishoeck, E. F., Blake, G. A., Jansen, D. J., \& Groesbeck, T. D. 1995, ApJ, 447, 760

Ward-Thompson, D.,André, P., Crutcher, R., Johnstone, D., Onishi, T., \& Wilson, C. 2007, in:

B. Reipurth, D. Jewitt, \& K. Keil (eds.), Protostars and Planets V, 33

\section{Discussion}

ZIURYS: So you've got this efficiency of organic formation increases in this hot core. Is that partly a temperature effect or evaporation of certain things from grains, or combination?

Ceccarelli: What we've seen in hot corinos is the end of the process. I think that a big role is played by the grains, via surface chemistry. First of all, the abundance of COMs is so elevated that gas phase reactions very likely are not efficient enough. We have this wonderful laboratory experiment that shows that every time there is recombination in 
the gas phase, the large ions break, they do not form. Thus, we don't know whether the processes are driven by cosmic rays, or because the molecules on the ices that began to be mobile as the dust is warming up.

ZIURYS: How about three body neutral reactions? Can the grains can be the third body?

CECCARELli: The densities in the hot corinos are large, but not large enough to have a 3- body reaction.

UNKNOWN: What is the energy source for the formation of these complex molecules? UV?

Ceccarelli: May be cosmic rays. I don't think it can be UV because UV radiation does not penetrate this kind of sources, unless it is secondary UV from cosmic rays.

UnKNOWN: So besides the complex molecules, would some tholins be formed?

IRVINE: It would be hard to observe them in gas phase.

Mumma: The whole issue of the fate of materials formed in corinos is unclear. You may still have accretion on the central star, or may be outward transport, as evidenced by crystalline silicates in comets. The issue of where the materials that we observe originated has to do with whether you can trade off evolution time in the dense cloud core by cosmic rays for more rapid ionization in young systems, e. g. high abundance of $\mathrm{H}_{3}^{+}$formed by X-rays. So I really doubt that we have yet a clear picture of the degree of which the chemistry of corinos relates to the chemistry that we might see in primitive Solar System materials.

CecCARelli: I agree that we do not have a clear picture yet, and deuteration can help us to understand what is the fate of these molecules. It is an imprint that we can use.

Mumma: I think that is exactly right, a testable hypothesis, if we can use the deuterated molecules, ortho/para ratios, and other cosmonautic parameters. 


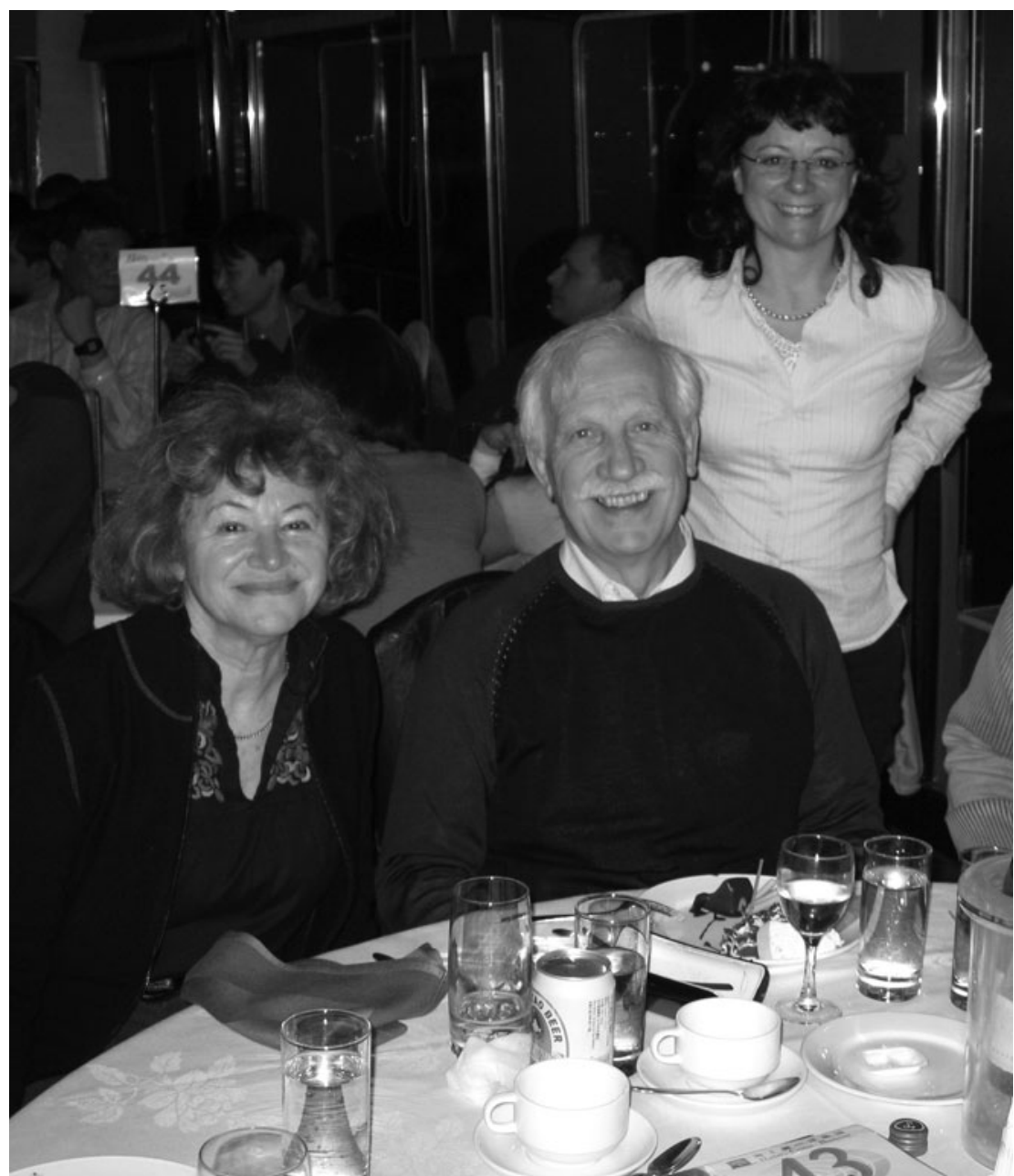

Catherine Cesarsky, Philippe Brechignac, and Cecilia Ceccarelli enjoying the buffet during the harbour cruise. 